\title{
Penetration of Wind-Generated Near-Inertial Waves into a Turbulent Ocean
}

\author{
Olivier AsSELIN AND WiLliam R. YOUNG \\ Scripps Institution of Oceanography, University of California, San Diego, La Jolla, California
}

(Manuscript received 23 December 2019, in final form 30 March 2020)

\begin{abstract}
An idealized storm scenario is examined in which a wind-generated inertial wave interacts with a turbulent baroclinic quasigeostrophic flow. The flow is initialized by spinning up an Eady model with a stratification profile based on observations. The storm is modeled as an initial value problem for a mixed layer confined, horizontally uniform inertial oscillation. The primordial inertial oscillation evolves according to the phaseaveraged model of Young and Ben Jelloul. Waves feed back onto the flow by modifying the potential vorticity. In the first few days, refraction dominates and wave energy is attracted (repelled) by regions of negative (positive) vorticity. Wave energy is subsequently drained down into the interior ocean guided by anticyclonic vortices. This drainage halts as wave energy encounters weakening vorticity. After a week or two, wave energy accumulates at the bottom of negative vorticity features, that is, along filamentary structures at shallow depths and in larger anticyclonic vortices at greater depths. Wave feedback tends to weaken vortices and thus slows the penetration of waves into the ocean interior. This nonlinear effect, however, is weak even for vigorous storms.
\end{abstract}

\section{Introduction}

Near-inertial waves comprise half of the energy and most of the vertical shear in the ocean internal wave spectrum (Ferrari and Wunsch 2009). These waves most strikingly manifest as near-circular surface drifter orbits (Poulain 1990; D'Asaro et al. 1995) and as a ubiquitous spectral peak in moored current-meter data (Webster 1968; Fu 1981; Alford et al. 2016). Because of their large vertical shear, near-inertial waves are thought to be major drivers of upper-ocean mixing (Gregg et al. 1986; Hebert and Moum 1994; Kunze et al. 1995).

Near-inertial waves originate in the ocean surface mixed layer with the 1000-km horizontal scale characteristic of atmospheric storms (Pollard 1980; Thomson and Huggett 1981; D'Asaro et al. 1995). Were these waves to preserve this large initial horizontal scale, they would never penetrate vertically or contribute to mixing below the mixed layer (Gill 1984). If the wave frequency is close to the Coriolis frequency $f$, then the vertical group velocity is

$$
c_{g}^{z} \approx \mathrm{Bu} f / m,
$$

where $m$ is the vertical wavenumber. The Burger number in (1) is $\mathrm{Bu}=(\mathrm{Nk} / \mathrm{fm})^{2}$, with $k$ the horizontal

Corresponding author: Olivier Asselin, oasselin@ucsd.edu wavenumber, and $N$ the buoyancy frequency. With $N \sim 10^{-2} \mathrm{~s}^{-1}$ and $f \sim 10^{-4} \mathrm{~s}^{-1}$ in the midlatitude pycnocline, $k^{-1} \sim 10^{6} \mathrm{~m}$ and $m^{-1} \sim 100 \mathrm{~m}$, the Burger number is of order $10^{-4}$. Thus, the time required to propagate vertically through a distance of $100 \mathrm{~m}$ is about 3 years. Unless there is a reduction of the horizontal scale from the $1000-\mathrm{km}$ generation scale, vertical propagation of near-inertial waves is glacially slow.

A main outcome of the Ocean Storms Experiment was observational evidence that the latitudinal variation of the Coriolis frequency-the $\beta$ effect-leads to a systematic reduction of the horizontal scale of near-inertial waves (D'Asaro et al. 1995). The primordial inertial wave oscillates at different frequencies in its southernmost and northernmost regions, which results in a dephasing of the initially uniform orbits. The dephasing increases with time and so produces an ever smaller meridional wavelength, resulting in significant vertical propagation of near-inertial waves (D'Asaro 1989).

These observations, however, were made in a region with weak mesoscale variability (D'Asaro 1995). In fact, atmospheric storm tracks (and thus near-inertial energy) largely coincide with regions of strong mesoscale variability (Zhai et al. 2005). In addition to the $\beta$ effect, mesoscale vorticity has long been hypothesized to cause local frequency shifts analogous to the $\beta$ effect (Mooers 1975a,b; Perkins 1976; Kunze 1985). These theoretical 
works predict that vertical vorticity of mesoscale and submesoscale eddies, $\zeta$, shifts the local inertial frequency by $\zeta / 2$. Gradients of eddy vorticity are at least an order of magnitude larger than the $\beta$ effect (Van Meurs 1998). Thus the $\zeta / 2$ frequency shift might be more important than $\beta$ in reducing horizontal scales and accelerating vertical propagation. Argo float data show that both the amplitude of the seasonal cycle of diapycnal mixing and the response to increases in the wind energy flux are larger in regions with an energetic eddy field (Whalen et al. 2012, 2018).

Theory predicts that the eddy field imprints its horizontal scale of roughly $10-100 \mathrm{~km}$ onto the wave field through refraction. However, early theoretical studies were largely based on the Wentzel-Kramers-Brillouin (WKB) assumption that the spatial scale of the waves is much less than that of the mesoscale flow. This scaleseparation assumption is strongly violated by freshly generated near-inertial waves. In this paper, we employ the model of Young and Ben Jelloul (1997, hereafter YBJ), which does not rely on the WKB approximation. Instead, YBJ relies on the timescale separation between the slow mesoscale and fast near-inertial waves, and makes use of phase averaging to remove the fast inertial oscillation and expose the slow evolution of the backrotated near-inertial wave velocity. YBJ also predicts that eddy vorticity shifts the local inertial frequency by a factor of $\zeta / 2$, confirming the WKB prediction.

The YBJ model was first employed to probe the enhanced propagation of near-inertial waves due to simple vorticity distributions (Balmforth et al. 1998), the $\beta$ effect (Moehlis and Llewellyn Smith 2001), or both simultaneously (Balmforth and Young 1999). Llewellyn Smith (1999) used the YBJ model to calculate the trapped near-inertial modes of a barotropic axisymmetric vortex. Klein and Llewellyn Smith (2001) and Klein et al. (2004) explored the dispersion of nearinertial waves by a fully turbulent barotropic quasigeostrophic flow. Danioux et al. (2008) employed a primitive equation model to study the three-dimensional propagation of wind-generated near-inertial waves. The behavior of shear-containing modes was successfully captured by the YBJ model. These authors also reported a deep maximum of vertical velocity with frequency $2 f$, further investigated by Danioux and Klein (2008), Danioux et al. (2011), and Wagner and Young (2016) extended the YBJ model by including the nonlinearly generated $2 f$-harmonic in the phase-averaged equations.

The original YBJ model describes the passive distortion of near-inertial waves due to advection and refraction by a balanced flow. Xie and Vanneste (2015) derived a fully coupled model in which strong nearinertial waves may feed back onto the balanced flow by modifying its potential vorticity; see also Wagner and Young $(2015,2016)$ and Salmon (2016). Rocha et al. (2018) employed the model of Xie and Vanneste (2015) to investigate energy exchanges between nearinertial waves and barotropic flows. In the absence of vertical shear, waves have a fixed vertical wavelength and the wave capture mechanism of Bühler and McIntyre (2005) does not function. Instead, waves propagate out of straining regions and this wave escape prevents efficient transfer of energy with the barotropic flow.

In this paper, we explore how turbulent eddies facilitate the penetration of wind-generated energy into the ocean interior. We consider an idealized storm scenario in which an inertial wave initially confined to the mixed layer interacts with turbulent baroclinic quasigeostrophic eddies. These eddies are initialized by spinning up an Eady model with a stratification profile based on observations (section 2). The subsequent coupled evolution of quasigeostrophic (QG) eddies and near-inertial waves (NIW) is represented with a novel QG-NIW model (section 3). Wave evolution is governed by the $\mathrm{YBJ}^{+}$model (Asselin and Young 2019), a higher-order version of the YBJ model. Waves also feed back onto the balanced flow by modifying the potential vorticity (Xie and Vanneste 2015). The simulations reported here are the first integrations of a YBJ-like system coupled with threedimensional baroclinic turbulence. The combination of a phase-averaged equation for the waves with quasigeostrophy provides a reduced description of vertical near-inertial propagation into geostrophic turbulence. The model captures all relevant physical processes, with the notable exception of wave breaking and turbulent mixing. The primitive equations are more realistic, but the wave-eddy decomposition is then challenging and ambiguous; the QG-NIW model has the advantage of representing waves and eddies with distinct variables so that the wave-eddy decomposition is hardwired. The QG-NIW model is an ideal platform to visualize both the propagation of waves in the wake of a storm (section 4) and the effects of waves on eddies (section 5). We assemble our findings into a coherent narrative in section 6 .

\section{Problem setup}

The numerical experiment has two phases: the flow spinup and the storm. The spinup generates a turbulent mesoscale flow using the Eady model of baroclinic instability generalized with an observation-based stratification profile. Once the Eady solution reaches statistical stationarity, Eady forcing terms are removed and the 

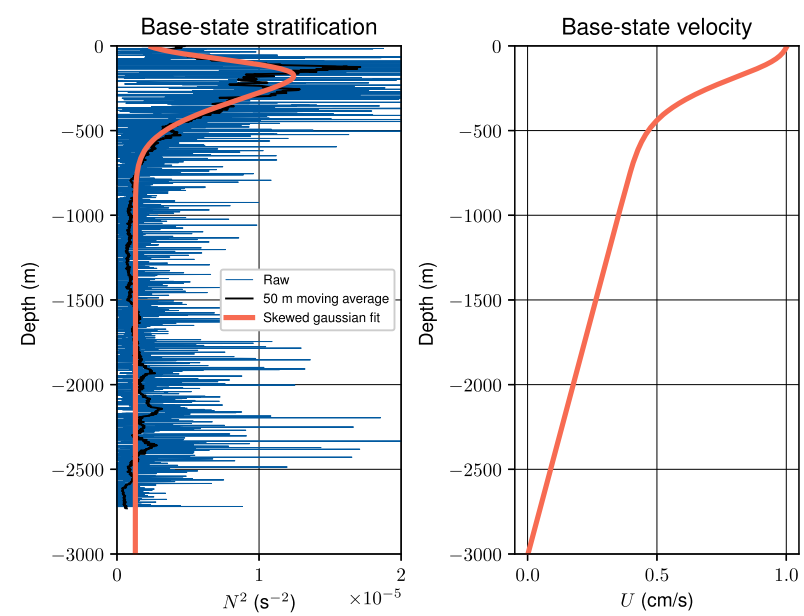

FIG. 1. (left) Typical raw stratification profile (blue) observed during the NISKINe pilot cruise, along with its $50-\mathrm{m}$ moving average (black). The orange line is the skewed Gaussian fit [(3)] with parameters outlined in Table 1. (right) Base-state zonal velocity profile ensuring zero potential vorticity gradient with the skewed Gaussian fit for stratification.

storm phase begins. The storm is modeled as an initial value problem for the wave field. The wave model is initialized with a horizontally uniform inertial oscillation in the mixed layer, which subsequently evolves in the mature geostrophic flow. This section describes the initial condition used for the storm experiment.

\section{a. Phase I: Setting up a turbulent mesoscale field}

A generalized Eady model (Eady 1949) is employed to generate a mesoscale eddy field. A steady vertically sheared geostrophic base flow is imposed

$$
\Psi=-U(z) y,
$$

where $\Psi$ and $U$ are the streamfunction and zonal velocity of the base state. Meridional and vertical components of the base-state velocity are zero. An Eady model is characterized by a vanishing base-state potential vorticity gradient. This is achieved if the vertical shear is proportional to the squared buoyancy frequency: $U^{\prime}(z) \propto N^{2}(z)$. The classic Eady model is the special case with constant $N^{2}$ and a linearly sheared base-state velocity. Here instead, we use observations from the Near-Inertial Shear and Kinetic Energy Experiment (NISKINe) pilot cruise to set $N^{2}$. NISKINe is at around $58.5^{\circ} \mathrm{N}$, so we use $f=1.24 \times 10^{-4} \mathrm{~s}^{-1}$.

Figure 1 shows the raw observations (blue), 50-m moving average (black) and fit (red). The observed profile is typical of the month of May in the subpolar gyre of the North Atlantic. There is a relatively wellmixed layer in top 50-100 m overlaying a thermocline
TABLE 1. Fitting parameters for $N^{2}$.

$\begin{array}{lrl}\text { Abyssal stratification } & N_{0}^{2}=1.2927 \times 10^{-6} \mathrm{~s}^{-2} \\ \text { Gaussian amplitude } & N_{1}^{2}=6.4532 \times 10^{-6} \mathrm{~s}^{-2} \\ \text { Gaussian location } & z_{0}=-77.1809 \mathrm{~m} \\ \text { Width parameter } & \sigma=309.6155 \mathrm{~m} \\ \text { Skewness parameter } & \alpha & =-5.3384\end{array}$

that extends to about $600-700 \mathrm{~m}$. Below the thermocline, the abyss has constant and relatively weak stratification down to the ocean bottom, located at depth of $3 \mathrm{~km}$.

We fit the smoothed observed $N^{2}(z)$ profile with

$$
N_{\text {fit }}^{2}=N_{0}^{2}+N_{1}^{2} e^{-\left(z-z_{0}\right)^{2} / \sigma^{2}}\left\{1+\operatorname{erf}\left[\frac{\alpha\left(z-z_{0}\right)}{\sigma \sqrt{2}}\right]\right\} .
$$

Table 1 displays the values used for the fit. The abyssal value of stratification $N_{0}^{2}$ is calculated as the average smoothed $N^{2}$ below $1 \mathrm{~km}$. The right panel of Fig. 1 shows the base-state velocity profile $U(z)$. The magnitude of velocity $U(z)$ (and corresponding shear) is a free parameter. We adjust the amplitude of $U(z)$ so that the equilibrated eddy field has the maximum sea surface eddy velocity of about $25 \mathrm{~cm} \mathrm{~s}^{-1}$ obtained from both in situ observations during the NISKINe cruise, and from geostrophic currents derived from satellite altimetry (Bonjean and Lagerloef 2002). It is remarkable that this level of eddy energy is produced with the small mean velocity difference $\Delta U=1 \mathrm{~cm} \mathrm{~s}^{-1}$ indicated in the right panel of Fig. 1.

The turbulent mesoscale field grows from quasigeostrophic baroclinic instability of the base flow $U(z)$. This growth is eventually halted by the bottom friction produced by a 60-m-thick Ekman bottom layer. The spinup ends once the eddy energy reaches statistical stationarity. At this point, we remove the energy-injecting base state in (2) to better isolate wave-eddy interactions.

Figure 2 displays the snapshots of the equilibrated mesoscale field used as an initial condition for the storm experiments. The surface flow is a realization of surface quasigeostrophic turbulence (Johnson 1978; Blumen 1978; Lapeyre 2017). Figure 2 shows secondary roll-up of filaments (Held et al. 1995). The left panel of Fig. 3 shows a shallow $k^{-2}$ kinetic energy spectrum at the sea surface (Pierrehumbert et al. 1994) which rapidly steepens and weakens with increasing depth (Smith and Bernard 2013; Asselin et al. 2016, 2018). In the bottom panels of Fig. 2, features with larger horizontal scales have deeper vertical penetration scales. This is consistent with surface quasigeostrophic dynamics: features with horizontal wavenumber $k$ decays exponentially with a vertical scale $f / N k$ (Tulloch and Smith 2006). 

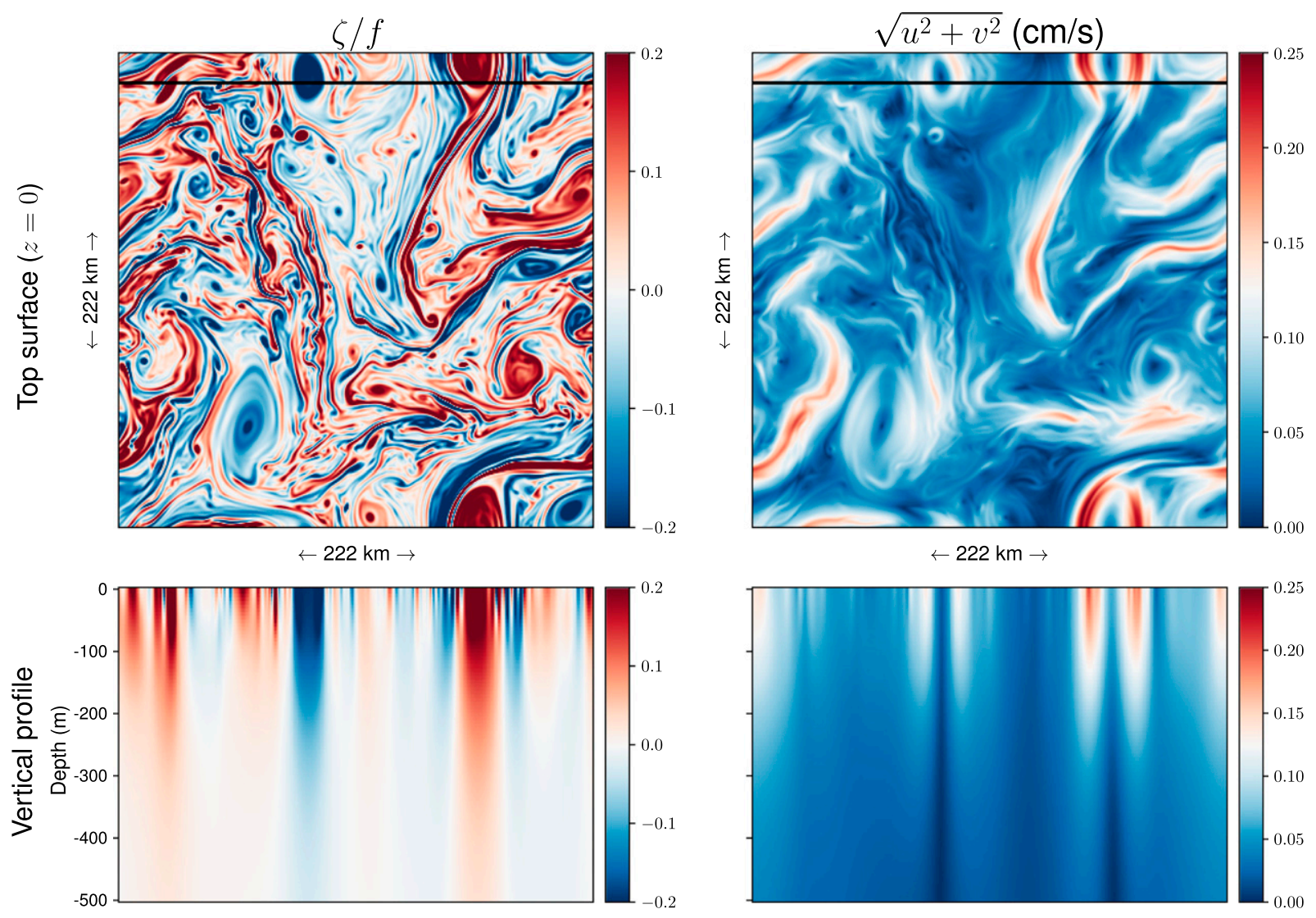

FIG. 2. Flow initial condition for the storm experiment. (left) Vertical relative vorticity normalized by the Coriolis frequency. (right) Magnitude of the geostrophic velocity. (top) Surface view. (bottom) An $x-z$ section of the top $500 \mathrm{~m}$ taken along the horizontal line drawn in the top panels.

The right panel of Fig. 3 shows the vertical profile of horizontally averaged energy and vorticity for the initial condition. The vorticity and velocity fields decay vertically within the first few hundred meters (only the top $500 \mathrm{~m}$ are shown, but the domain is $3 \mathrm{~km}$ deep). The vorticity-based root-mean-square (rms) Rossby number, Ro $=\zeta_{\text {rms }} / f$, reaches up to about 0.14 at the surface, comparable with the value of 0.10 in the simulations of Danioux et al. (2008).

\section{b. Phase II: Setting up a storm}

Pollard and Millard (1970) found strong agreement between inertial currents from moored observations and those calculated from a simple slab model of the mixed layer. In this slab model, the mixed layer behaves as a harmonic oscillator with a resonant frequency $f$ (neglecting the artificial damping term). Midlatitude storms typically have a strong inertial component (D'Asaro 1985) and thus cause the mixed layer to resonate at its natural frequency.

In our idealized scenario, we assume that a passing storm impulsively excites an inertial oscillation throughout the mixed layer without influencing the geostrophic flow. Such events have been reported during the Oceans Storms Experiment-see, for instance, the second storm analyzed in Dohan and Davis (2011). Atmospheric storms have horizontal scales on the order of $1000 \mathrm{~km}$, so we shall assume that the windgenerated inertial oscillation is horizontally uniform in our $222 \mathrm{~km} \times 222 \mathrm{~km}$ domain (about $2^{\circ}$ of longitude squared). As a result, this idealized scenario can be translated into an initial value problem for the wave field, with an initial condition that is a horizontally uniform unidirectional current confined to the mixed layer:

$$
u=u_{0} e^{-z^{2} / \sigma_{w}^{2}}, \quad v=w=b=0,
$$

where $u, v, w$, and $b$ are the wave velocities and buoyancy. The parameter $\sigma_{w}$ is a proxy for the mixed layer depth. The initial wave field is given by (4) with $\sigma_{w}=50 \mathrm{~m}$ : see the red curve of the right panel of Fig. 3. The storm strength is represented by the surface velocity $u_{0}$. Unless otherwise specified, we use the value $u_{0}=10 \mathrm{~cm} \mathrm{~s}^{-1}$. The impact of storm strength is 

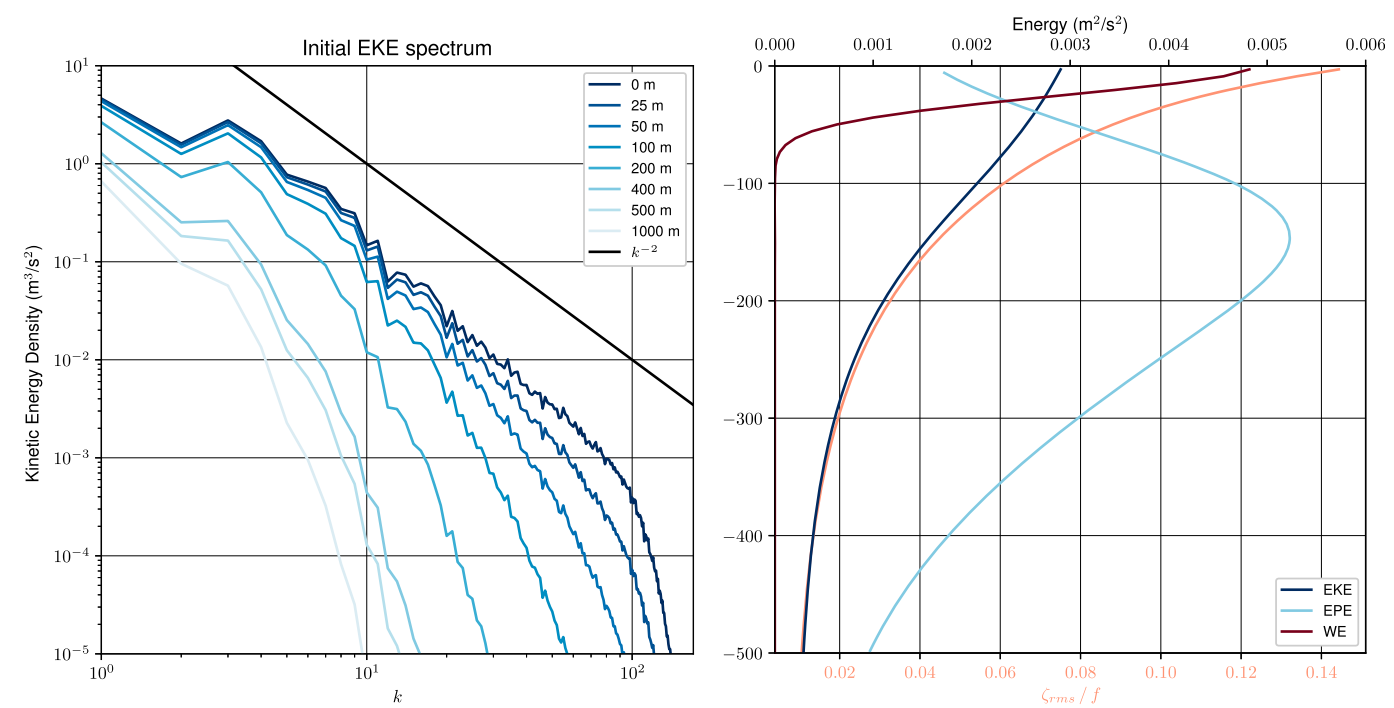

FIG. 3. Initial condition for the simulations. (left) Horizontal wavenumber eddy kinetic energy spectrum at various depths. (right) Vertical profile of eddy kinetic (EKE) and potential (EPE) energy, wave energy (WE, using $u_{0}=10 \mathrm{~cm} \mathrm{~s}^{-1}$ ), and normalized rms vorticity.

covered in section 5 , where we consider values up to $u_{0}=40 \mathrm{~cm} \mathrm{~s}^{-1}$ as well as a control storm with no wave feedback, which is equivalent to $u_{0} \rightarrow 0$.

\section{The QG-NIW model}

The previous section described the initial condition used for eddy and wave fields; here we discuss their subsequent evolution. In essence, the QG-NIW model couples the quasigeostrophic equation with the $\mathrm{YBJ}^{+}$ equation (Asselin and Young 2019) for near-inertial waves:

$$
\begin{gathered}
\partial_{t} q+J\left(\psi^{\mathrm{L}}, q\right)=\mathscr{D}_{q}, \\
\partial_{t} \mathrm{~L}^{+} A+J\left(\psi^{\mathrm{L}}, \mathrm{L}^{+} A\right)+\frac{i}{2} \triangle \psi^{\mathrm{L}} \mathrm{L}^{+} A+\frac{i f}{2} \triangle A=\mathscr{D}_{\mathrm{L}^{+} A},
\end{gathered}
$$

where $i=\sqrt{-1}$ is the imaginary unit, $J(a, b)=a_{x} b_{y}-a_{y} b_{x}$ is the horizontal Jacobian, $\triangle=\partial_{x}^{2}+\partial_{y}^{2}$ is the horizontal Laplacian, and $\mathrm{L}^{+}$is a frequently occurring operator defined by

$$
\mathrm{L}^{+} \stackrel{\text { def }}{=} \mathrm{L}+\frac{1}{4} \triangle, \quad \text { where } \quad \mathrm{L} \stackrel{\text { def }}{=} \frac{\partial}{\partial z}\left(\frac{f^{2}}{N^{2}} \frac{\partial}{\partial z}\right) .
$$

The complex field $A$ relates to the back-rotated velocity of the near-inertial wave field-see (8) below-while $\psi^{\mathrm{L}}$ and $q$ are the streamfunction and potential vorticity of the balanced flow. The superscript $\mathrm{L}$ emphasizes that $\psi^{\mathrm{L}}$ is a streamfunction for the Lagrangian-mean flow, that is, the potential vorticity $q$ and wave envelope $\mathrm{L}^{+} A$ are advected by both the Eulerian-mean flow and the Stokes drift associated with near-inertial waves. Finally, $\mathscr{D}$ represents small-scale dissipative processes, detailed in (19) and (20) below.

\section{a. Wave evolution}

Wave evolution is dictated by the $\mathrm{YBJ}^{+}$equation (6). Reading terms from left to right, $\mathrm{YBJ}^{+}$describes the evolution of the near-inertial wave envelope due to advection, refraction, dispersion and dissipation. In this framework, the horizontal wave velocities, $u$ and $v$, are combined into a single complex field $A(x, y, z, t)$ with the fast inertial rotation removed,

$$
\mathrm{L} A \stackrel{\mathrm{def}}{=}(u+i v) e^{i f t} .
$$

Since waves are assumed near-inertial, the wave envelope (8) evolves slowly compared with the inertial period. In the original YBJ model, this wave envelope constitutes the prognostic variable from which all wave fields can be derived (like $q$ in the QG system). In $\mathrm{YBJ}^{+}$ the prognostic variable employs the improved operator $\mathrm{L}^{+}$instead of the original $L$ in (7). This tweak in the definition of the wave envelope brings the twin advantages of higher accuracy and lower computational effort while maintaining ease of implementation (Asselin and Young 2019).

\section{b. Flow evolution}

The evolution of the balanced flow is dictated by (5), which is identical to the traditional quasigeostrophic potential vorticity equation (Charney 1948; Salmon 1998; Vallis 2017). Here, however, $\psi^{\mathrm{L}}$ and $q$ are defined as the 
streamfunction and potential vorticity of the Lagrangianmean balanced flow:

$$
q=\triangle \psi^{\mathrm{L}}+\mathrm{L} \psi^{\mathrm{L}}+\underbrace{\frac{i}{2 f} J\left(\mathrm{~L}^{+} A^{*}, \mathrm{~L}^{+} A\right)+\frac{1}{4 f} \triangle\left|\mathrm{L}^{+} A\right|^{2},}_{q^{w}}
$$

where $A^{*}$ is the complex conjugate of $A$. The first two terms on the right-hand side of (9) are relative vorticity and vortex stretching. The other term, $q^{w}$, is the wave potential vorticity, or the wave feedback onto the flow. This term was first derived using the generalizedLagrangian-mean framework (Xie and Vanneste 2015) before enjoying independent confirmations via multipletimescale asymptotic expansion of the Eulerian primitive equations (Wagner and Young 2015), and via an analogy with the variational principle for classical electrodynamics (Salmon 2016). The wave potential vorticity $q^{w}$ is quadratic in wave amplitude; in the limit of weak waves the standard quasigeostrophic approximation is recovered. Wagner and Young (2015) emphasize that the quadratic terms in $q^{w}$ arise from nonlinearities thrown up by phase-averaged potential vorticity dynamics. Near-inertial waves are special because all nonlinearities in $q^{w}$ are concisely expressed in terms of the back-rotated velocity, as in (9).

The difference between the Lagrangian-mean and Eulerian-mean flow variables-the Stokes correctionscales like Ro times the wave-to-flow kinetic energy ratio. The magnitude of this Stokes correction can be estimated by inspecting Fig. 3 , in which $\zeta_{\mathrm{rms}} / f$ is a Rossby number. With the standard value $u_{0}=10 \mathrm{~cm} \mathrm{~s}^{-1}$, we obtain a Stokes correction on the order of $20 \%$. This indicates that the Eulerian-mean and Lagrangian-mean definitions do not differ qualitatively: flow variables may be interpreted as typical Eulerian-mean quantities for our standard runs (all of section 4). For storm strengths of $20 \mathrm{~cm} \mathrm{~s}^{-1}$ or more, however, the Stokes "correction" may be as large as its Eulerian-mean and Lagrangian-mean counterparts. We consider these strong storms in section 5 .

\section{c. Conservation laws}

In this section we summarize the two conservation laws of the QG-NIW system (5), (6), and (9); see Asselin and Young (2019) for more details. All phase-averaged descriptions of wave dynamics conserve wave action (Bretherton and Garrett 1968) and $\mathrm{YBJ}^{+}$is no exception: multiplying the inviscid version of $\mathrm{YBJ}^{+}$equation (6) with $\mathrm{L}^{+} A^{*}$, adding the complex conjugate, and performing a volume average, one finds

$$
\frac{d}{d t}\langle\mathrm{WE}\rangle=0
$$

where the brackets \langle\rangle denote volume averaging, and we introduced the wave energy density

$$
\mathrm{WE} \stackrel{\text { def }}{=} \frac{1}{2}\left|\mathrm{~L}^{+} A\right|^{2} .
$$

The action density is $\mathrm{WE} / f$, where the divisor $f$ is the intrinsic frequency of near-inertial waves. We prefer to use WE as our main index of near-inertial wave activity.

$\mathrm{WE}$ is a high-order approximation to the energy density of near-inertial waves. Specifically, the volume average of WE can be decomposed as

$$
\langle\mathrm{WE}\rangle=\underbrace{\left\langle\mathrm{WKE}_{0}\right\rangle}_{\mathrm{Bu}^{0}}+\underbrace{\langle\mathrm{WPE}\rangle}_{\mathrm{Bu}^{1}}+\underbrace{\left\langle\mathrm{WKE}^{+}\right\rangle}_{\mathrm{Bu}^{2}},
$$

where

$$
\begin{gathered}
\mathrm{WKE}_{0} \stackrel{\text { def }}{=} \frac{1}{2}|\mathrm{~L} A|^{2}, \\
\mathrm{WPE} \stackrel{\text { def }}{=} \frac{1}{4} \frac{f^{2}}{N^{2}}\left|\nabla A_{z}\right|^{2}, \\
\mathrm{WKE}^{+} \stackrel{\text { def }}{=} \frac{1}{32}|\triangle A|^{2} .
\end{gathered}
$$

The order of magnitude of each contribution in terms of the Burger number, $\mathrm{Bu}$, is indicated in the underbraces in (12). The first and largest term on the right of (12) is a leading-order approximation to the kinetic energy of the near-inertial waves. The second term is the wave potential energy, which is smaller than wave kinetic energy by a factor of $\mathrm{Bu} \ll 1$. The final, and smallest, term on the right of (12) is a higher-order correction to the wave kinetic energy.

Coupling $\mathrm{YBJ}^{+}$with the QG system yields a second invariant, coupled energy,

$$
\frac{d}{d t}\left\langle\mathrm{WCE}+\mathrm{EE}^{\mathrm{L}}\right\rangle=0,
$$

where we introduced the wave part of coupled energy,

$$
\mathrm{WCE} \stackrel{\text { def }}{=} \mathrm{WPE}+2 \mathrm{WKE}^{+},
$$

and the total Lagrangian-mean eddy energy:

$$
\mathrm{EE}^{\mathrm{L}} \stackrel{\text { def }}{=} \frac{1}{2}\left|\nabla \psi^{\mathrm{L}}\right|^{2}+\frac{1}{2} \frac{f^{2}}{N^{2}}\left|\psi_{z}^{\mathrm{L}}\right|^{2}
$$

The existence of this second conservation law is expected: the original Boussinesq system conserves energy, and so too must the wave-averaged system (Xie and Vanneste 2015). The physical interpretation of 
the coupled energy is complicated because $\mathrm{EE}^{\mathrm{L}}$ is based on Lagrangian-mean, not Eulerian-mean velocity and buoyancy. Because of Stokes corrections, $\mathrm{EE}^{\mathrm{L}}$ is not easily related to the kinetic and potential energy of the parent Boussinesq model. The interpretation of QGNIW energetics in terms of familiar Eulerian-mean quantities is the subject of ongoing research; see Rocha et al. (2018) for a discussion of the problem.

In our storm scenario, waves are initially uniform such that the wave part of coupled energy WCE, which depends on gradients in the wave field, is zero. Creation of horizontal gradients in the wave field thus inevitably leads to loss of Lagrangian-mean eddy energy $\mathrm{EE}^{\mathrm{L}}$. This is a manifestation of stimulated generation, a process whereby waves initially present in the balanced flow cause it to lose Lagrangian-mean energy $\mathrm{EE}^{\mathrm{L}}$ (Xie and Vanneste 2015; Rocha et al. 2018).

\section{d. Numerical details}

The QG and $\mathrm{YBJ}^{+}$equations (5) and (6) are solved using the same numerical methods. Both are pseudospectral in the $x$ and $y$ directions, allowing horizontal derivatives to be computed with spectral accuracy. The 2/3 rule is used to remove aliased modes (Durran 2013). Vertical derivatives are approximated with second-order centered finite differences. The resolution used is $512^{3}$. Since the domain is $222 \times 222 \mathrm{~km}^{2}$ in the horizontal and $3 \mathrm{~km}$ deep, this gives $\Delta x \approx 433 \mathrm{~m}$ and $\Delta z \approx 6 \mathrm{~m}$, uniformly spaced. Time integration is accomplished with the leapfrog scheme with weak time diffusion (Asselin 1972). Dissipation occurs through horizontal hyperdiffusion:

$$
\begin{aligned}
\mathscr{D}_{q} & =\nu_{1} \triangle^{6} q+\nu_{2} \triangle^{2} q, \\
\mathscr{D}_{\mathrm{L}^{+} A} & =\nu_{1} \triangle^{6} \mathrm{~L}^{+} A,
\end{aligned}
$$

with $\nu_{1}=3.2 \times 10^{25} \mathrm{~m}^{12} \mathrm{~s}^{-1}$ and $\nu_{2}=9.5 \times 10^{3} \mathrm{~m}^{4} \mathrm{~s}^{-1}$.

\section{Wave propagation}

We begin by looking at how eddies distort the wave field and allow penetration into the ocean interior. We found that the main storyline is not qualitatively affected by the storm strength. This section thus focusses on the standard case with wave feedback resulting from an initial near-inertial velocity $u_{0}=10 \mathrm{~cm} \mathrm{~s}^{-1}$. We postpone discussion of $u_{0}$ dependence and wave feedback onto eddies to section 5 .

\section{a. Early refractive phase}

Figure 4 shows snapshots of sea surface wave energy (WE) and normalized vertical vorticity, $\zeta / f=\Delta \psi^{\mathrm{L}} / f$, after 1,2, 5, 10 and 20 days (from top to bottom). The eye is immediately arrested by the rapid imprinting of the vorticity scales onto the wave field, leading to a collapse of the near-inertial wave horizontal scale.

This early refractive phase has been studied by Klein et al. (2004). For an initially pure inertial wave (horizontally uniform amplitude and phase), the small-time balance in (6) is

$$
\partial_{t} \mathrm{~L} A \approx-\frac{i}{2} \zeta \mathrm{L} A,
$$

where next-order $\mathrm{YBJ}^{+}$corrections are omitted for convenience. The early-time wave solution is therefore:

$$
\mathrm{L} A \approx \mathrm{L} A_{0} e^{-i \zeta t / 2},
$$

where the 0 subscript denotes the horizontally uniform initial condition in (4). Let us decompose the complex wave envelope in terms of real-valued amplitude $R$ and phase $\theta: L A=R e^{i \theta}$. Taking the absolute value of (22) indicates that refraction leaves $R$ unchanged. Instead, at early times, refraction causes a shift in the wave phase:

$$
\theta \approx \theta_{0}-\frac{1}{2} \zeta t
$$

Using altimetry and surface drifter data, Elipot et al. (2010) found that phase shifts approximately follow $-0.39 \zeta t$, which is reasonably near to the refractive shift predicted here [and by Kunze (1985) and others].

To obtain the evolution of the wave scale, one takes the horizontal gradient of (23),

$$
\mathbf{k} \stackrel{\text { def }}{=} \nabla \theta \approx-\frac{1}{2} \nabla \zeta t
$$

which is the expression obtained by Kunze (1985). Thus, refraction leads to the reduction of the wave horizontal scale: this is the essential ingredient that increases the vertical group velocity resulting in propagation. Including dispersive effects, Klein et al. (2004) further showed that the early-time amplitude of the nearinertial wave distributes like the Laplacian of vorticity; see also Klein and Treguier (1995). Note however that $\triangle \zeta$ is strongly anticorrelated with $\zeta$ (Elipot et al. 2010), which explains the strong anticorrelation between wave energy and vorticity evident at 1 day and 2 days in Fig. 4.

This attraction of wave energy into anticyclonic regions, and repulsion from cyclonic regions, has been observed repeatedly (Weller 1985; Kunze 1986; Kunze et al. 1995; Elipot et al. 2010). Explanations have been proposed relying on the broadening of the allowable frequency band in negative vorticity regions and subsequent trapping of rays (Kunze 1985), appeals to the quantum analogy between energy wells and negative 


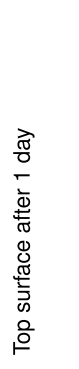
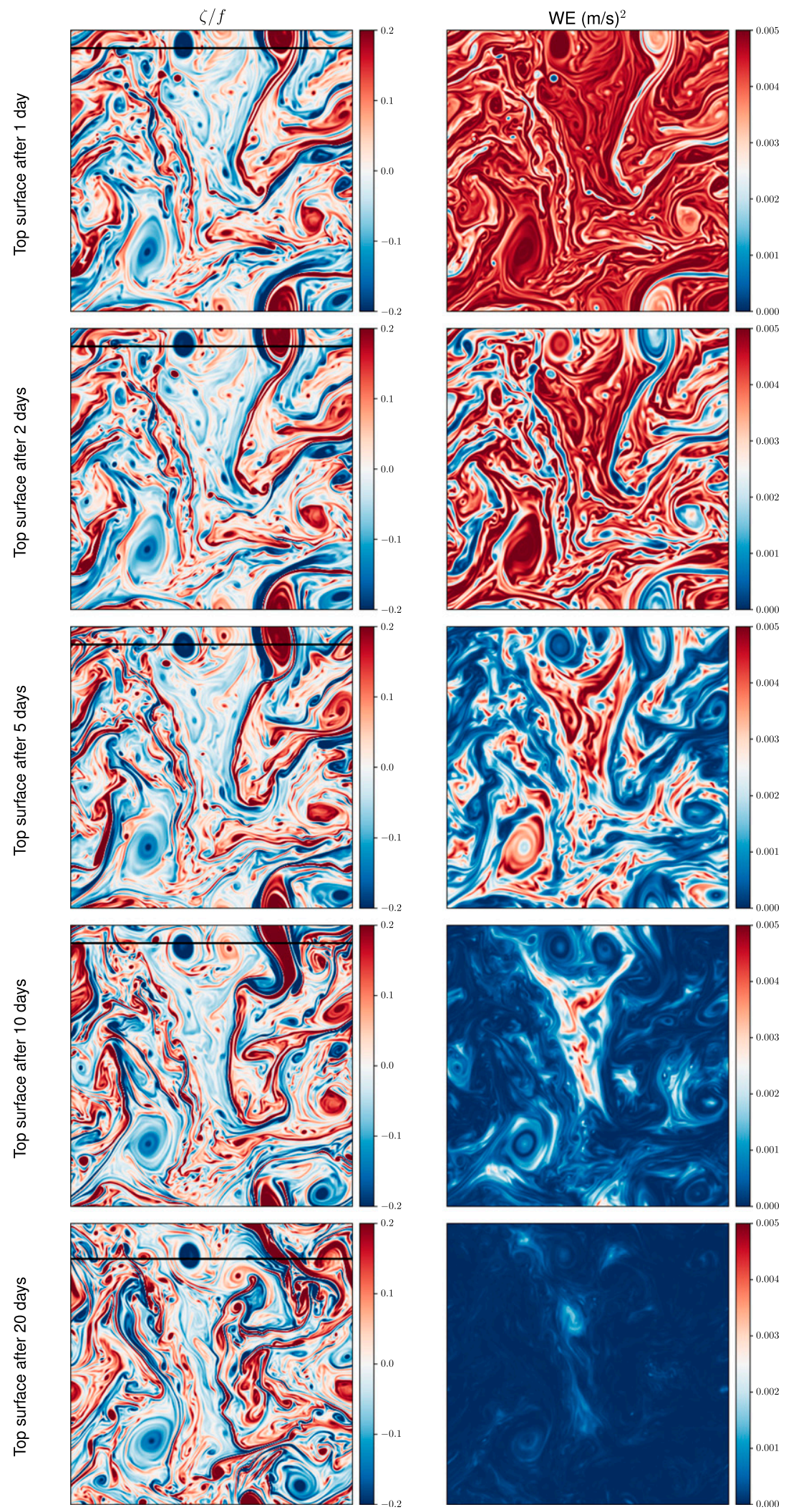

FIG. 4. Time series of normalized vertical vorticity, $\zeta / f=\Delta \psi^{\mathrm{L}} / f$, and wave energy density at the sea surface. Horizontal lines in the vorticity panels indicate the location of the vertical slices in Fig. 6. 
vorticity (Balmforth et al. 1998), and more recently, a conservation law of the YBJ model that applies to nearinertial waves in a steady flows (Danioux et al. 2015).

\section{b. Later-time wave energy-vorticity correlation}

Sea surface wave energy is repelled from cyclones and attracted by anticyclones during the early-time refractive phase. At longer times, however, the story is more complicated. For instance, pick the negative (blue) and positive (red) vortices transected by the black line in Fig. 4 . In the early stage ( 1 and 2 days) these vortices are associated with high (in the anticyclone) and low (in the cyclone) concentrations of WE. But after 5 days, WE is gone from the negative vortex whereas the positive vortex has drawn in some filaments of WE. This is a result of vertical propagation: the WE initially focused into the negative vortices has fluxed downward (further discussion below). At 10 days, the small amount of WE that remains at the sea surface is mostly in the region of weak vorticity.

Figure 5 quantifies this evolution by displaying the average WE in different bins of surface vorticity, from strong negative to strong positive (see the right panel for the vorticity distribution and bin definitions). Early on, the more negative surface vorticity is, the more WE concentrates in this region. For the intermediate and strong positive regions there is a rapid decline of energy. After about 5 days, it is regions of weaker vorticity (and intermediate negative) that retain most of the sea surface WE; see also the bottom panels of Fig. 4. These weak-vorticity regions are also characterized by weak vorticity gradients which, according to (24), are associated with a slower reduction of horizontal scale and thus slower vertical propagation.

Using inverse excess bandwidth of near-inertial peaks in global drifter data, Elipot et al. (2010) estimated the average decay time of near-inertial waves at the surface as a function of the flow vorticity. Consistent with our simulations (in particular, Fig. 4), they found that decay time is maximum for weaker vorticity, and decreases faster with increasing positive vorticity than negative vorticity.

Elipot et al. (2010) also report that, on average, more surface wave energy populates anticyclonic than cyclonic regions. But the difference is small-about a factor of 2 between the strongest positive and negative vorticity regions, with a relatively flat response for weaker vorticity (see their Fig. 15). This is consistent with a time average of the $\zeta-W E$ correlation in Fig. 5. Although the refractive phase initially causes a strong concentration of WE into anticyclones-WE in strong negative vortices is up to a factor of 5 larger than WE in strong positive vortices-the time-averaged surface correlation is rapidly diluted by vertical propagation of waves into the ocean interior. We speculate that in statistical steady state, in which near-inertial waves are intermittently forced by the passage of storms, the distribution of WE will depend on the frequency of storms that reinitiate the early-time refractive phase at the sea surface. In general, we expect the timeaveraged surface $\zeta$-WE correlation to be less than predicted by $\zeta$ refraction alone.

\section{c. Inertial drainpipes}

A main message of Fig. 4 is that WE vanishes rapidly from the sea surface. Most of this surface loss is because WE radiates into the cean interior. Figure 6 displays an $x-z$ section of WE at a fixed $y$ along the black line overlaid on the vorticity plots in Fig. 4; contours are overlaid to indicate regions of positive (solid) and negative (dashed) vorticity.

The picture is clear: WE leaves regions of positive vorticity within a day or two. This transfer is mostly lateral (Kunze 1985; Balmforth et al. 1998; Lee and Niiler 1998). After 2 days, there is significant downward propagation of energy along the cores of anticyclonic (negative) vortices. Almost no subsurface WE is found in cyclonic vortices. Anticyclones are waveguides that drain WE downward into the deeper ocean (Kunze 1985; Balmforth et al. 1998; Lee and Niiler 1998; Zhai et al. 2005; Danioux et al. 2008). After 10 or 20 days, WE accumulates at the bottom of anticyclones. Using ray tracing, Kunze (1985) predicted this trapping of waves at the bottom of anticyclones as they encounter a critical layer-a region defined by vanishing vertical wavelength and group velocity as vorticity weakens. Wave trapping at the base of anticyclones has frequently been observed (Kunze 1986; Kunze et al. 1995; Oey et al. 2008; Joyce et al. 2013; Martínez-Marrero et al. 2019; Kawaguchi et al. 2019).

Figure 7 shows horizontal cuts of $\zeta$ and WE at depths of 50,100, and $200 \mathrm{~m}$ after 10 days. As seen in Fig. 2, vorticity features with larger horizontal scales penetrate to greater depths. Like $\zeta$, WE is seen in larger-scale features at greater depths. Unlike $\zeta$, however, largescale WE features are not seen at shallow depths: this is because WE collects at the bottom of anticyclones. For instance, at $50 \mathrm{~m}$, WE is only evident in the smallest vortices and filaments. At $100 \mathrm{~m}, \mathrm{WE}$ is found only in intermediate-scale features, and at $200 \mathrm{~m}$, WE only occupies large anticyclonic cores. These observations provide further evidence that waves get trapped at critical layers at the base of vortices.

\section{d. Mixing}

As near-inertial waves propagate into the ocean interior, their vertical shear may come to exceed the 

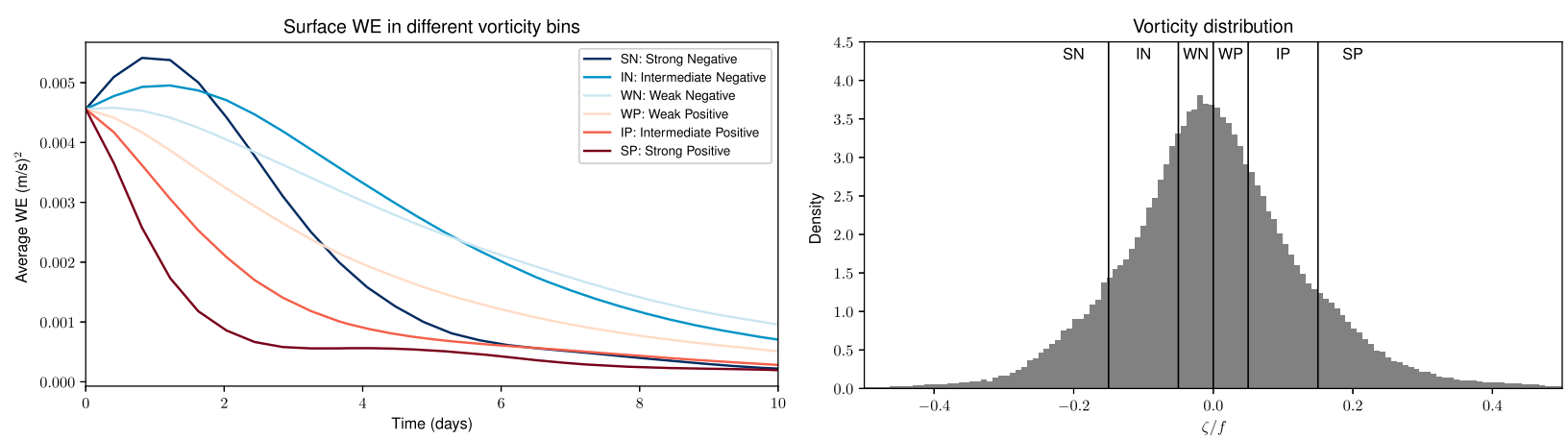

FIG. 5. (left) Time series of the average of surface WE in regions of strong, intermediate, and weak positive/negative vorticity. (right) Initial distribution of vorticity (undergoes little change over time period shown). Boundaries between bins are defined as $\zeta / f=0, \pm 0.05$, and \pm 0.015 .

stabilization provided by background stratification. In this case waves may break through shear instabilities and cause mixing. But the phase-averaged wave model filters out these instabilities (YBJ). The necessary condition for instability may nevertheless be diagnosed from model output.

Figure 8 shows vertical cuts of inverse Richardson number,

$$
\mathrm{Ri}^{-1} \stackrel{\operatorname{def}}{=} \frac{1}{2} \frac{u_{z}^{2}+v_{z}^{2}}{N^{2}}
$$

which quantifies the relative stabilizing and destabilizing contributions of stratification and vertical shear. Values of 4 or more (saturated red) indicate that shear instabilities could occur, and thus provides a proxy for mixing.

During first 10 days, unstable conditions are mostly confined to the top $100 \mathrm{~m}$, in spite of significant wave energy present at greater depths (compare with Fig. 6). This is because stratification is weaker near the surface (Fig. 1). After 20 days, unstable conditions extend throughout the whole depth of the large negative vortex. Overall, unstable conditions are collocated with wave energy in anticyclones, echoing the observations of Lueck and Osborn (1986) and Kunze et al. (1995).

\section{e. Depth distribution of wave energy}

So far, we have seen that wave energy rapidly leaves the sea surface (Fig. 4), and localized vertical cuts revealed that at least some of this wave energy flows downward through anticyclonic drainpipes (Fig. 6). We conclude this section by quantifying the horizontally averaged depth distribution of wave energy.

The left panel of Fig. 9 shows the time evolution of the depth partition of wave energy. Following the wave initial condition, (4), over $90 \%$ of WE is initially located in the upper $100 \mathrm{~m}$. This energy is, however, rapidly drained to depth. After 20 days, the upper $100 \mathrm{~m}$ retains about only $20 \%$ of the WE, as is typical in observations (D'Asaro et al. 1995; Elipot et al. 2010). Most of the energy remains in the top kilometer with about $5 \%$ in each of the bottom two kilometers.

Recall from (10) that total wave energy WE is conserved in the absence of forcing and dissipative processes. In this initial value problem, loss of WE (top white region) may thus only occur through dissipation operating at small horizontal wave scales. After one month of evolution, about $20 \%$ of WE is dissipated. This contrasts with the $<1 \%$ dissipative rates found in the barotropic simulations of Rocha et al. (2018). In a barotropic flow, the vertical wavelength is fixed and straining increases the wave group velocity. Waves thus escape straining regions. In our baroclinic simulations, however, the vertical wavelength is not fixed and wave escape may be less effective. Over longer time scales, wave energy slowly radiates vertically. Equipartition between the top, middle, and bottom kilometer bins requires about half a year of evolution (not shown). By that time, about $70 \%$ of WE is dissipated.

The right panel of Fig. 9 provides a more detailed view of the WE vertical profile in the top $500 \mathrm{~m}$. Again, WE rapidly drains out of the mixed layer, but vertical propagation stops at around $200 \mathrm{~m}$, a depth comparable to that of the strongest vortices of the eddy field (see bottom panels of Fig. 2). This generalizes the intuition obtained from localized vertical cuts (Fig. 6): WE is drained down to the base of anticyclonic vortices.

\section{Wave feedback}

So far we have seen that eddies distort the primordial wind-generated inertial oscillation and guide wave energy downward into the ocean interior via anticyclonic drainpipes. In this simple scenario, waves play a passive role: eddies are indifferent to the presence of 

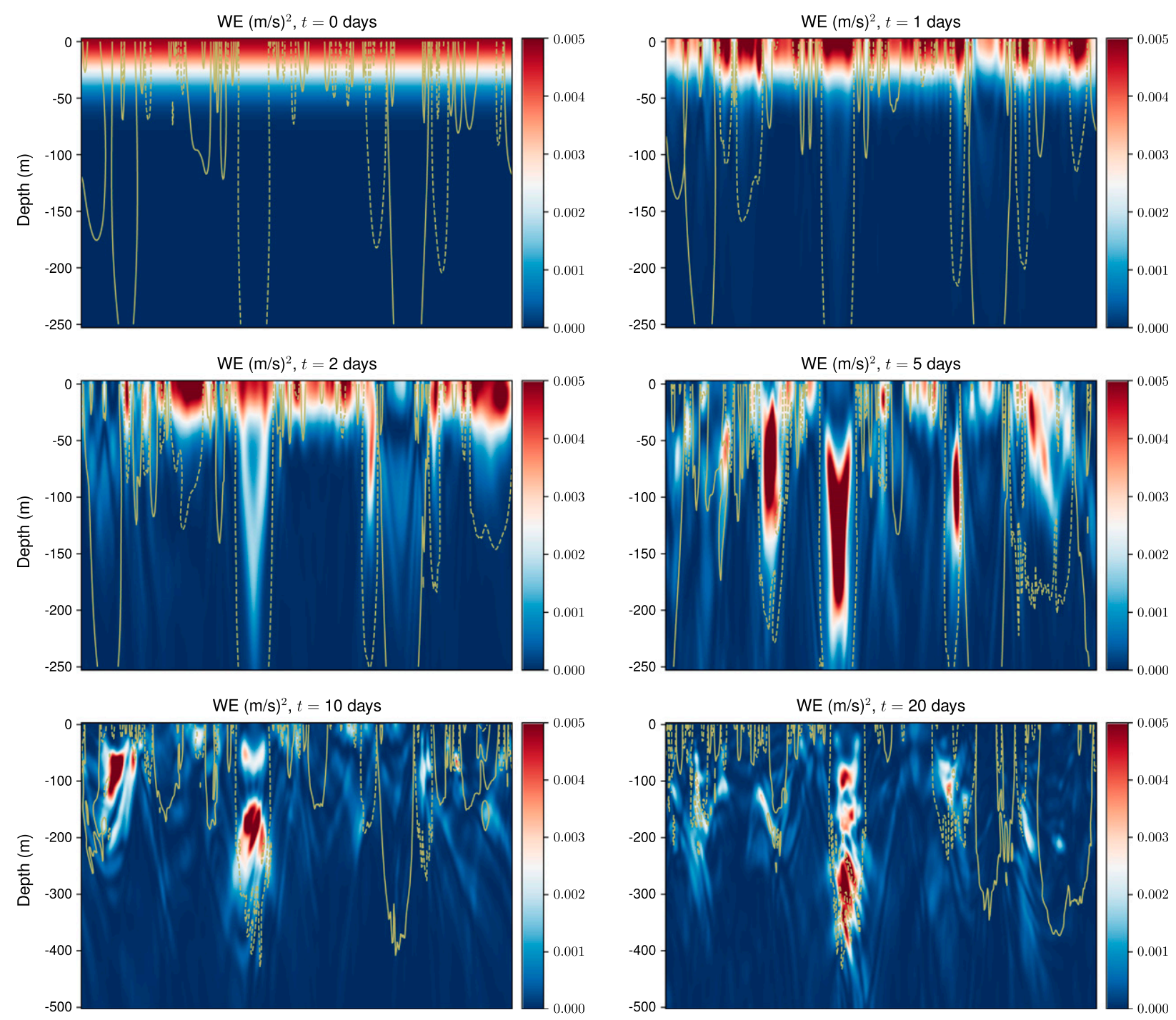

FIG. 6. The $x-z$ snapshots of WE with overlaid contours of vorticity (solid: $\zeta / f=0.05$, dashed: $\zeta / f=-0.05$ ). The top $250 \mathrm{~m}$ are shown for the 5 first days, then the top $500 \mathrm{~m}$ are shown. The whole $x$ extent of the domain is shown. The $y$ location of slices is indicated by horizontal lines in the vorticity distributions of Fig. 4.

waves. The QG-NIW model, however, includes the wave feedback $q^{w}$ in (9). Does this wave feedback mechanism play a role?

The answer depends on the wind strength. In all simulations above, we have taken the initial wave velocity $u_{0}=10 \mathrm{~cm} \mathrm{~s}^{-1}$. In this case, although waves and eddies are equally strong, it turns out that wave feedback onto eddies is weak. The top panels of Fig. 10 display sea surface vertical vorticity and WE in a noninteracting control run $\left(q^{w}=0\right)$; the middle panels show the standard case with wave feedback and $u_{0}=$ $10 \mathrm{~cm} \mathrm{~s}^{-1}$. Although sea surface WE is slightly larger in the simulation with feedback, the distribution of WE looks qualitatively similar with and without feedback.
Moreover, there is no perceptible difference in the vorticity field between the two cases. This indicates that that wave feedback is negligible at the sea surface if $u_{0}=10 \mathrm{~cm} \mathrm{~s}^{-1}$.

Wave feedback becomes more apparent if the initial wave velocity is increased to $40 \mathrm{~cm} \mathrm{~s}^{-1}$ (bottom panels of Fig. 10). Because $q^{w}$ is quadratic in the wave amplitude, this increases the wave feedback by a factor of 16. In this strong-wave case, the vorticity field is noticeably different: finescale features appear and some of the intermediate-scale features are smoothed out, especially in anticyclonic regions. Most strikingly, the relative vorticity is, on the whole, weakened by wave feedback. 

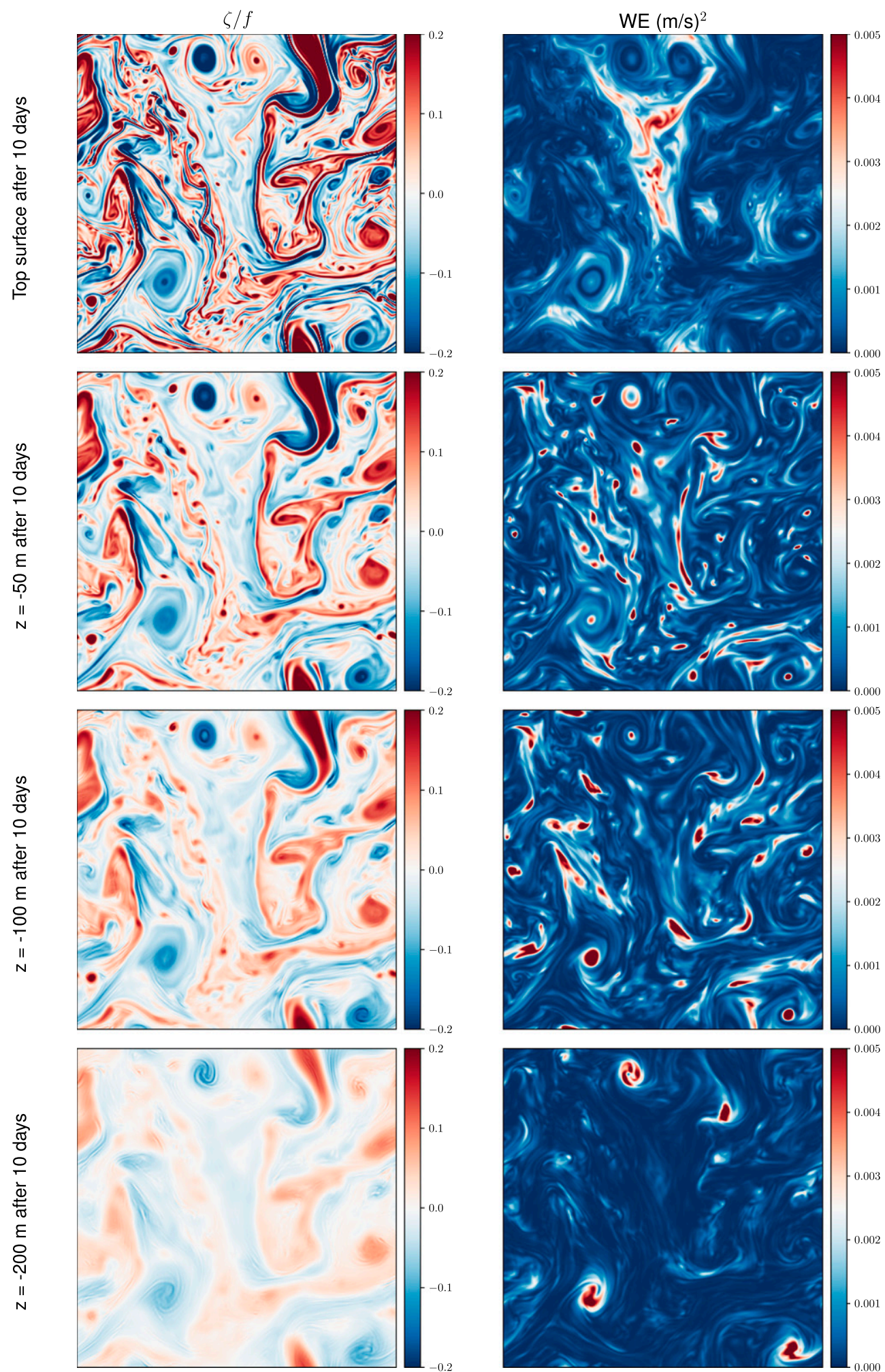

FIG. 7. Normalized vertical vorticity, $\zeta / f=\Delta \psi^{\mathrm{L}} / f$, and wave energy density after 10 days at various depths. 

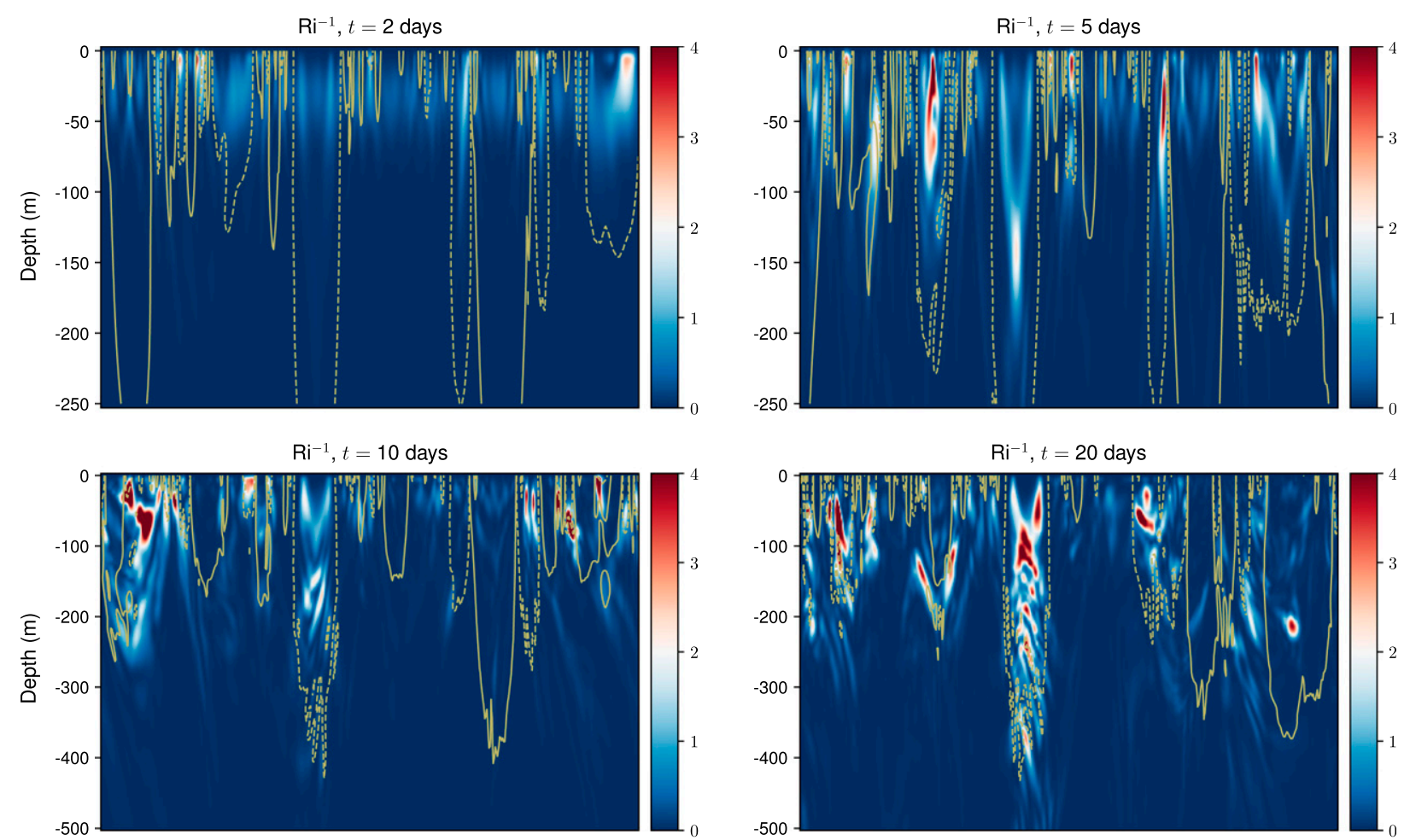

FIG. 8. The $x-z$ snapshots of the inverse Richardson number with overlaid contours of vorticity (solid: $\zeta / f=0.05$, dashed: $\zeta / f=-0.05$ ). Values over 4 indicate potential shear instability. The top $250 \mathrm{~m}$ are shown for the 5 first days, then the top $500 \mathrm{~m}$ are shown.

This is a manifestation of stimulated loss of balance, or stimulated generation (Xie and Vanneste 2015; Rocha et al. 2018). This mechanism is best described using the coupled energy conservation law (16), which indicates that the sum of total Lagrangian-mean eddy energy, $\mathrm{EE}^{\mathrm{L}}$, and the wave part of coupled energy, WCE, is a conserved quantity. Recall from (17) that WCE, which is approximately equal to wave potential energy, depends on horizontal gradients in the wave field. Since our simulations are initialized with a horizontally uniform inertial oscillation, WCE is initially zero. As waves are refracted and strained by the flow, gradients

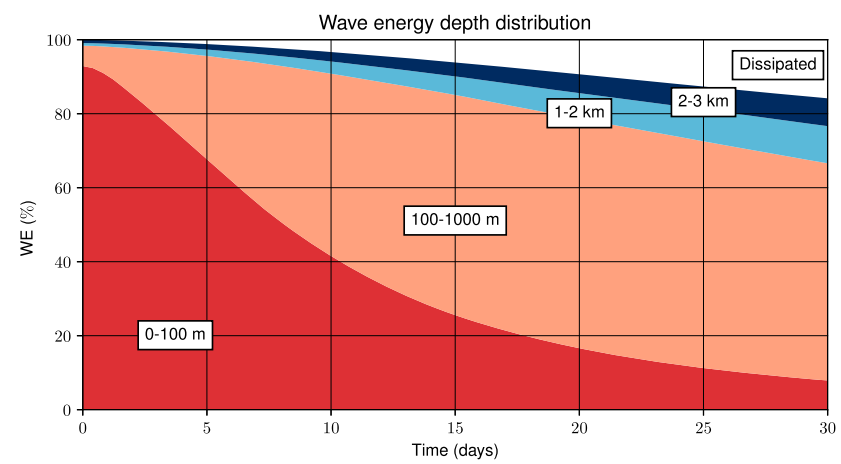

emerge in the wave field and WCE increases. The coupled energy conservation law dictates that the growth of WCE ineluctably entails a decrease of $\mathrm{EE}^{\mathrm{L}}$. This explains why strong wave feedback weakens the Lagrangian-mean eddies in the bottom-left panel of Fig. 10.

As a result of the dampening of balanced eddies, refraction is weaker. Following (24), wave scales are reduced more gently. Because the vertical wave group velocity (1) is proportional to the horizontal wavenumber squared, wave penetration is slower. Consequently, more wave energy remains at the surface after 10 days in the $40 \mathrm{~cm} \mathrm{~s}^{-1}$ storm (bottom-right

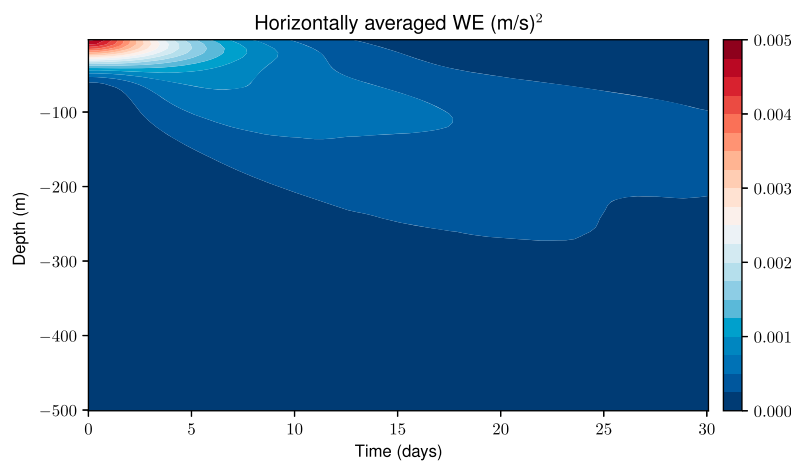

FIG. 9. (left) Depth partition of total wave energy (WE) as a function of time. The white region is energy dissipated by horizontal hyperviscosity. (right) WE density distribution in the top $500 \mathrm{~m}$. 

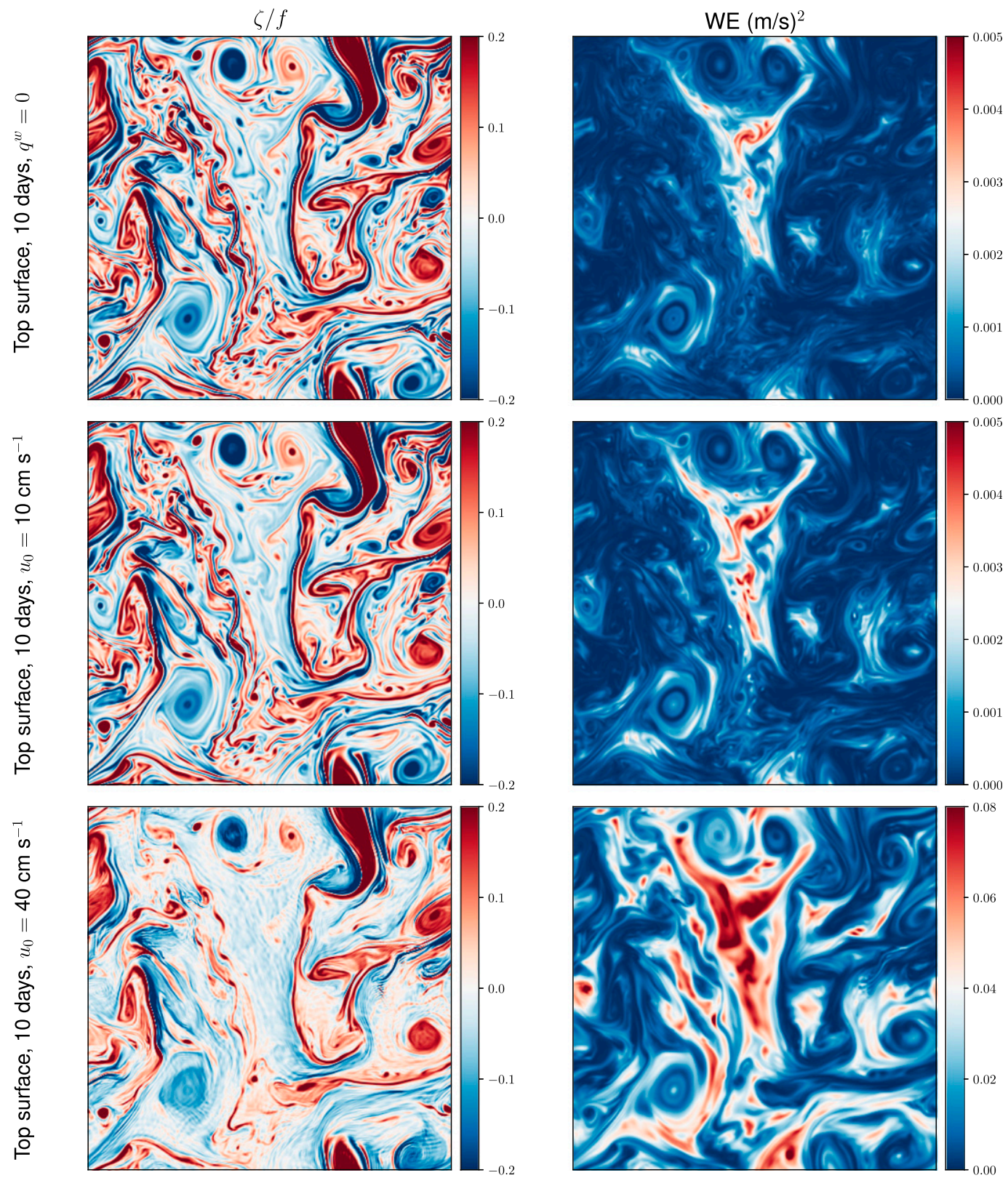

FIG. 10. Normalized surface vorticity, $\zeta / f=\Delta \psi^{\mathrm{L}} / f$, and WE after 10 days. (top) No feedback $\left(q^{w}=0\right)$. In this case the magnitude of $u_{0}$ is arbitrary. To facilitate comparison, we set $u_{0}=10 \mathrm{~cm} \mathrm{~s}^{-1}$. (middle) Standard storm with wave feedback and $u_{0}=10 \mathrm{~cm} \mathrm{~s}^{-1}$. (bottom) Strong storm with wave feedback and $u_{0}=40 \mathrm{~cm} \mathrm{~s}^{-1}$. The wave energy color bar is rescaled by a factor of 16 for comparison with the two top panels.

panel of Fig. 10). Wave penetration is faster without wave feedback (top-right panel of Fig. 10).

Wave feedback is also visible at the bottom of anticyclones, where wave energy collects and eddies weaken. Upon careful examination of the vorticity cuts of Fig. 7, which are from the standard $10 \mathrm{~cm} \mathrm{~s}^{-1}$ run after 10 days, one notes the appearance of small-scale "grooves" with increasing depth. These grooves are collocated with accumulations of wave energy and are absent from simulations with $q^{w}=0$ (not shown).

Figure 11 breaks down potential vorticity, $q$ in (9), into three constituents-relative vorticity, $\zeta=\Delta \psi^{\mathrm{L}}$, stretching, $L \psi^{\mathrm{L}}$, and wave potential vorticity, $q^{w}$. Figure 11 is at the same time and location as the lowest panels of Fig. 7. In the Eady model studied here, $q=0$ in the interior and so 

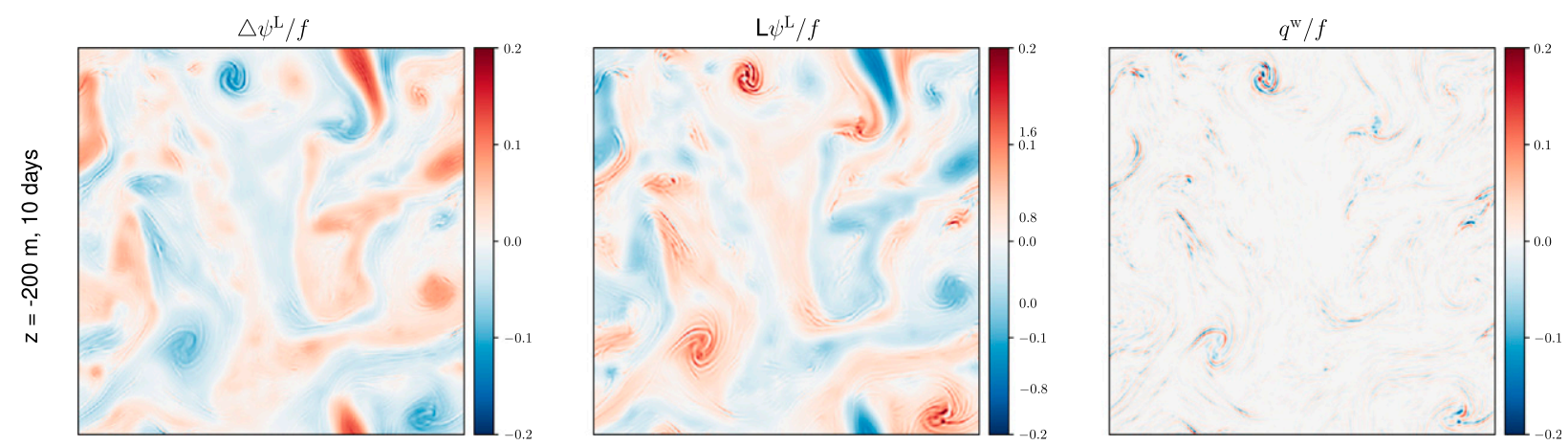

FIG. 11. Breakdown of potential vorticity $q$ defined in (9), for the standard run with $u_{0}=10 \mathrm{~cm} \mathrm{~s}^{-1}$, shown at $z=200 \mathrm{~m}$ after 10 days. In the Eady problem, $q=0$ everywhere in the interior, implying that $\triangle \psi^{\mathrm{L}}+\mathrm{L} \psi^{\mathrm{L}}+q^{w}=0$.

$$
\triangle \psi^{\mathrm{L}}+\mathrm{L} \psi^{\mathrm{L}}+q^{w}=0
$$

at every interior point and for all time. To satisfy (26), the fields shown in the three panels of Fig. 11 sum to zero. Thus as the wave field develops horizontal gradients, $q^{w}$ becomes nonzero, and these changes must be compensated by relative vorticity and stretching to maintain (26). Because $q^{w}$ is composed of high-order derivatives of the wave field, it magnifies the small-scale features of the wave field. It is the necessary cancellation of these small-scale $q^{w}$ features that produces grooves in the relative vorticity and stretching fields in the first two panels of Fig. 11.

These arguments are not limited to the Eady model, for which $q$ is zero. Even if $q$ is nonzero, QG dynamics dictates that $q$ is conserved (5). At any point in space and time, changes imposed by waves through $q^{w}$ must be canceled by relative vorticity and stretching to preserve the material invariance of $q$. A similar cancellation is seen in the $q \neq 0$ turbulent simulations of Rocha et al. (2018).

Despite these interesting features, wave feedback has, on the whole, a weak effect on wave propagation. When horizontal averages are performed, the depth partition of wave energy in Fig. 9 is qualitatively unchanged, even for the $40 \mathrm{~cm} \mathrm{~s}^{-1}$ strong-wave case. Sufficiently strong near-inertial waves do affect smallscale structure of eddies through $q^{w}$, but not strongly enough to significantly alter the vertical propagation of the waves.

\section{Discussion}

We have explored the penetration of wind-generated near-inertial energy into a baroclinically turbulent ocean using a new three-dimensional QG-NIW model. Let us now weave our findings into a narrative of the fate of near-inertial waves in the wake of a storm.
First, atmospheric storms with an appreciable inertial component (anticyclonic rotation near the local Coriolis frequency) sweep over the ocean, and the imparted momentum rapidly homogenizes in the mixed layer, leading to a primordial slab-like inertial oscillation (Pollard and Millard 1970; Dohan and Davis 2011). This initial wave has a horizontal scale comparable to that of the storm, and thus its initial vertical propagation is glacially slow (Gill 1984). During the first few days, however, $\zeta$ refraction leads to a collapse of the wave scales onto that of the vorticity field (Klein et al. 2004). Wave energy then fluxes into anticyclonic regions (Danioux et al. 2015). Anticyclones act as wave drains, guiding wave energy and shear downward into the ocean interior (Lee and Niiler 1998), likely leading to mixing (Kunze et al. 1995). These near-inertial drains terminate as the surface-intensified baroclinic vortices weaken at depth (Kunze 1985). Since the penetration depth of mesoscale structures is proportional to their horizontal scale (Lapeyre 2017), larger vortices penetrate deeper than smaller-scale filaments (Fig. 2). Horizontal cuts thus reveal small-scale wave energy patches at shallow depths, and larger-scale patches with increasing depth (Fig. 7).

Strong waves can weaken the Lagrangian-mean balanced eddies in which they are embedded through stimulated loss of balance (Xie and Vanneste 2015; Rocha et al. 2018). This in turn weakens refraction, which is responsible for the collapse of the primordial wave horizontal scale. Thus, wave feedback slows down the penetration of near-inertial waves into the ocean interior. That said, even for the most vigorous storm considered-corresponding to surface wave energy an order of magnitude larger than that of the balanced flow-the effect on wave propagation is weak. Given the numerical burden imposed by the high-derivative $q^{w}$ term, the uncoupled QG-NIW model is a preferred option for efficiently examining wave propagation. 
In the simulations described here, wind-generated near-inertial waves radiate out of the mixed layer on a time scale of a week or two: see Fig. 9. After a month, the bulk ( $\sim 60 \%)$ of wave energy is between 100 and $1000 \mathrm{~m}$ (corresponding to the termination depth of the vortices) while $20 \%$ has escaped to below $1-\mathrm{km}$ depth. We suspect, however, that these simulations overestimate the amount of wave energy penetrating the ocean interior. In these computations wave energy dissipates only through horizontal hyperdiffusion (20). The QG-NIW model of section 3 does not represent shear instabilities unless a parameterization is supplied (not the case here). Shear instabilities would provide a sink of wave energy via turbulent mixing (Lueck and Osborn 1986; Kunze et al. 1995). In fact, several investigators argue that a significant amount of nearinertial wave energy is lost to such turbulent mixing in the mixed layer (Zhai et al. 2009; Jochum et al. 2013; Soares and Richards 2013).

Acknowledgments. This work was supported by the National Science Foundation Award OCE-1657041 and by the Office of Naval Research Award N00014-18-12803. We are grateful for computer resources provided by the Extreme Science and Engineering Discovery Environment (XSEDE), which is supported by National Science Foundation Grant ACI-1548562. We thank Anna Savage for providing density profiles observed during the NISKINe pilot cruise.

\section{REFERENCES}

Alford, M. H., J. A. MacKinnon, H. L. Simmons, and J. D. Nash, 2016: Near-inertial internal gravity waves in the ocean. Annu. Rev. Mar. Sci., 8, 95-123, https://doi.org/10.1146/annurevmarine-010814-015746.

Asselin, O., and W. R. Young, 2019: An improved model of nearinertial wave dynamics. J. Fluid Mech., 876, 428-448, https:// doi.org/10.1017/jfm.2019.557.

— - P. Bartello, and D. N. Straub, 2016: On quasigeostrophic dynamics near the tropopause. Phys. Fluids, 28, 026601, https://doi.org/10.1063/1.4941761.

$\longrightarrow,-$, and 2018: On Boussinesq dynamics near the tropopause. J. Atmos. Sci., 75, 571-585, https://doi.org/10.1175/ JAS-D-17-0097.1.

Asselin, R., 1972: Frequency filter for time integrations. Mon. Wea. Rev., 100, 487-490, https://doi.org/10.1175/1520-0493(1972) $100<0487$ :FFFTI $>2.3$.CO;2.

Balmforth, N. J., and W. R. Young, 1999: Radiative damping of near-inertial oscillations in the mixed layer. J. Mar. Res., 57, 561-584, https://doi.org/10.1357/002224099321549594.

__, S. G. Llewellyn Smith, and W. R. Young, 1998: Enhanced dispersion of near-inertial waves in an idealized geostrophic flow. J. Mar. Res., 56, 1-40, https://doi.org/10.1357/ 002224098321836091.

Blumen, W., 1978: Uniform potential vorticity flow: Part I. Theory of wave interactions and two-dimensional turbulence. J. Atmos. Sci.,
35, 774-783, https://doi.org/10.1175/1520-0469(1978)035<0774: UPVFPI $>2.0 . \mathrm{CO} ; 2$.

Bonjean, F., and G. S. Lagerloef, 2002: Diagnostic model and analysis of the surface currents in the tropical Pacific Ocean. J. Phys. Oceanogr., 32, 2938-2954, https://doi.org/10.1175/ 1520-0485(2002)032<2938:DMAAOT>2.0.CO;2.

Bretherton, F. P., and C. J. R. Garrett, 1968: Wavetrains in inhomogeneous moving media. Proc. Roy. Soc. London, A302, 529-554, https://doi.org/10.1098/RSPA.1968.0034.

Bühler, O., and M. E. McIntyre, 2005: Wave capture and wavevortex duality. J. Fluid Mech., 534, 67-95, https://doi.org/ 10.1017/S0022112005004374.

Charney, J. G., 1948: On the scale of atmospheric motion. Geofys. Publ., 17 (2), 1-17.

Danioux, E., and P. Klein, 2008: A resonance mechanism leading to wind-forced motions with a $2 \mathrm{f}$ frequency. J. Phys. Oceanogr., 38, 2322-2329, https://doi.org/10.1175/2008JPO3822.1.

- - — , and P. Rivière, 2008: Propagation of wind energy into the deep ocean through a fully turbulent mesoscale eddy field. J. Phys. Oceanogr., 38, 2224-2241, https://doi.org/10.1175/2008JPO3821.1.

,$- \ldots$, M. W. Hecht, N. Komori, G. Roullet, and S. Le Gentil, 2011: Emergence of wind-driven near-inertial waves in the deep ocean triggered by small-scale eddy vorticity structures. $J$. Phys. Oceanogr., 41, 1297-1307, https://doi.org/10.1175/2011JPO4537.1.

_ J. Vanneste, and O. Bühler, 2015: On the concentration of near-inertial waves in anticyclones. J. Fluid Mech., 773, R2, https://doi.org/10.1017/JFM.2015.252.

D'Asaro, E. A., 1985: The energy flux from the wind to near-inertial motions in the surface mixed layer. J. Phys. Oceanogr., 15 , 1043-1059, https://doi.org/10.1175/1520-0485(1985)015<1043: TEFFTW $>2.0 . \mathrm{CO} ; 2$.

- 1989: The decay of wind-forced mixed layer inertial oscillations due to the $\beta$ effect. J. Geophys. Res., 94, 2045-2056, https://doi.org/10.1029/JC094iC02p02045.

_ 1995 : Upper-ocean inertial currents forced by a strong storm. Part III: Interaction of inertial currents and mesoscale eddies. J. Phys. Oceanogr., 25, 2953-2958, https://doi.org/10.1175/ 1520-0485(1995)025<2953:UOICFB > 2.0.CO;2.

— C. C. Eriksen, M. D. Levine, C. A. Paulson, P. Niiler, and P. Van Meurs, 1995: Upper-ocean inertial currents forced by a strong storm. Part I: Data and comparisons with linear theory. J. Phys. Oceanogr., 25, 2909-2936, https://doi.org/10.1175/ 1520-0485(1995)025<2909:UOICFB > 2.0.CO;2.

Dohan, K., and R. E. Davis, 2011: Mixing in the transition layer during two storm events. J. Phys. Oceanogr., 41, 42-66, https:// doi.org/10.1175/2010JPO4253.1.

Durran, D. R., 2013: Numerical Methods for Wave Equations in Geophysical Fluid Dynamics. Texts in Applied Mathematics, Vol. 32, Springer, 466 pp.

Eady, E. T., 1949: Long waves and cyclone waves. Tellus, 1, 33-52, https://doi.org/10.3402/tellusa.v1i3.8507.

Elipot, S., R. Lumpkin, and G. Prieto, 2010: Modification of inertial oscillations by the mesoscale eddy field. J. Geophys. Res., $\mathbf{1 1 5}$ C09010, https://doi.org/10.1029/2009JC005679.

Ferrari, R., and C. Wunsch, 2009: Ocean circulation kinetic energy: Reservoirs, sources, and sinks. Annu. Rev. Fluid. Mech., 41, 253-282, https://doi.org/10.1146/annurev.fluid.40.111406.102139.

Fu, L.-L., 1981: Observations and models of inertial waves in the deep ocean. Rev. Geophys., 19, 141-170, https://doi.org/ 10.1029/RG019i001p00141.

Gill, A., 1984: On the behavior of internal waves in the wakes of storms. J. Phys. Oceanogr., 14, 1129-1151, https://doi.org/ 10.1175/1520-0485(1984)014<1129:OTBOIW>2.0.CO;2. 
Gregg, M., E. D'Asaro, T. Shay, and N. Larson, 1986: Observations of persistent mixing and near-inertial internal waves. J. Phys. Oceanogr., 16, 856-885, https://doi.org/10.1175/1520-0485(1986) $016<0856$ :OOPMAN $>2.0 . \mathrm{CO} ; 2$.

Hebert, D., and J. Moum, 1994: Decay of a near-inertial wave. J. Phys. Oceanogr., 24, 2334-2351, https://doi.org/10.1175/ 1520-0485(1994)024<2334:DOANIW >2.0.CO;2.

Held, I. M., R. T. Pierrehumbert, S. T. Garner, and K. L. Swanson, 1995: Surface quasi-geostrophic dynamics. J. Fluid Mech., 282, 1-20, https://doi.org/10.1017/S0022112095000012.

Jochum, M., B. P. Briegleb, G. Danabasoglu, W. G. Large, N. J. Norton, S. R. Jayne, M. H. Alford, and F. O. Bryan, 2013: The impact of oceanic near-inertial waves on climate. J. Climate, 26, 2833-2844, https://doi.org/10.1175/JCLI-D12-00181.1.

Johnson, E. R., 1978: Topographically bound vortices. Geophys. Astrophys. Fluid Dyn., 11, 61-71, https://doi.org/10.1080/ 03091927808242652.

Joyce, T. M., J. M. Toole, P. Klein, and L. N. Thomas, 2013: A near-inertial mode observed within a Gulf Stream warmcore ring. J. Geophys. Res. Oceans, 118, 1797-1806, https:// doi.org/10.1002/jgrc.20141.

Kawaguchi, Y., T. Wagawa, and Y. Igeta, 2019: Near-inertial internal waves and multiple-inertial oscillations trapped by negative vorticity anomaly in the central sea of Japan. Prog. Oceanogr., 181, 102240, https://doi.org/10.1016/J.POCEAN.2019.102240.

Klein, P., and A. Treguier, 1995: Dispersion of wind-induced inertial waves by a barotropic jet. J. Mar. Res., 53, 1-22, https:// doi.org/10.1357/0022240953213331.

_ , and S. G. Llewellyn Smith, 2001: Horizontal dispersion of nearinertial oscillations in a turbulent mesoscale eddy field. J. Mar. Res., 59, 697-723, https://doi.org/10.1357/002224001762674908.

_,- , and G. Lapeyre, 2004: Organization of near-inertial energy by an eddy field. Quart. J. Roy. Meteor. Soc., 130,11531166, https://doi.org/10.1256/QJ.02.231.

Kunze, E., 1985: Near-inertial wave propagation in geostrophic shear. J. Phys. Oceanogr., 15, 544-565, https://doi.org/10.1175/ 1520-0485(1985)015<0544:NIWPIG > 2.0.CO;2.

_ 1986: The mean and near-inertial velocity fields in a warmcore ring. J. Phys. Oceanogr., 16, 1444-1461, https://doi.org/ 10.1175/1520-0485(1986)016<1444:TMANIV >2.0.CO;2.

_, R. W. Schmitt, and J. M. Toole, 1995: The energy balance in a warm-core ring's near-inertial critical layer. J. Phys. Oceanogr., 25, 942-957, https://doi.org/10.1175/1520-0485(1995)025<0942: TEBIAW $>2.0 . \mathrm{CO} ; 2$.

Lapeyre, G., 2017: Surface quasi-geostrophy. Fluids, 2, 7, https:// doi.org/10.3390/fluids2010007.

Lee, D.-K., and P. P. Niiler, 1998: The inertial chimney: The near-inertial energy drainage from the ocean surface to the deep layer. J. Geophys. Res., 103, 7579-7591, https://doi.org/10.1029/97JC03200.

Llewellyn Smith, S. G., 1999: Near-inertial oscillations of a barotropic vortex: Trapped modes and time evolution. J. Phys. Oceanogr., 29, 747-761, https://doi.org/10.1175/1520-0485(1999) $029<0747$ :NIOOAB $>2.0 . \mathrm{CO} ; 2$.

Lueck, R., and T. Osborn, 1986: The dissipation of kinetic energy in a warm-core ring. J. Geophys. Res., 91, 803-818, https:// doi.org/10.1029/JC091iC01p00803.

Martínez-Marrero, A., and Coauthors, 2019: Near-inertial wave trapping near the base of an anticyclonic mesoscale eddy under normal atmospheric conditions. J. Geophys. Res. Oceans, 124, 8455-8467, https://doi.org/10.1029/2019JC015168.

Moehlis, J., and S. G. Llewellyn Smith, 2001: Radiation of mixed layer near-inertial oscillations into the ocean interior. J. Phys.
Oceanogr., 31, 1550-1560, https://doi.org/10.1175/1520-0485(2001) 031<1550:ROMLNI $>2.0 . \mathrm{CO} ; 2$.

Mooers, C. N., 1975a: Several effects of a baroclinic current on the cross-stream propagation of inertial-internal waves. Geophys. Astrophys. Fluid Dyn., 6, 245-275, https://doi.org/10.1080/ 03091927509365797.

_ 1975b: Several effects of baroclinic currents on the threedimensional propagation of inertial-internal waves. Geophys. Astrophys. Fluid Dyn., 6, 277-284, https://doi.org/10.1080/ 03091927509365798.

Oey, L.-Y., M. Inoue, R. Lai, X.-H. Lin, S. Welsh, and L. Rouse Jr., 2008: Stalling of near-inertial waves in a cyclone. Geophys. Res. Lett., 35, L12604, https://doi.org/10.1029/2008GL034273.

Perkins, H., 1976: Observed effect of an eddy on inertial oscillations. Deep-Sea Res. Oceanogr. Abstr., 23, 1037-1042, https:// doi.org/10.1016/0011-7471(76)90879-2.

Pierrehumbert, R. T., I. M. Held, and K. L. Swanson, 1994: Spectra of local and nonlocal two-dimensional turbulence. Chaos, Solitons Fractals, 4, 1111-1116, https://doi.org/10.1016/09600779(94)90140-6.

Pollard, R. T., 1980: Properties of near-surface inertial oscillations. J. Phys. Oceanogr., 10, 385-398, https://doi.org/10.1175/15200485(1980)010<0385:PONSIO > 2.0.CO;2.

— , and R. Millard, 1970: Comparison between observed and simulated wind-generated inertial oscillations. Deep-Sea Res. Oceanogr. Abstr., 17, 813-821, https://doi.org/10.1016/00117471(70)90043-4.

Poulain, P.-M., 1990: Near-inertial and diurnal motions in the trajectories of mixed layer drifters. J. Mar. Res., 48, 793-823, https://doi.org/10.1357/002224090784988728.

Rocha, C. B., G. L. Wagner, and W. R. Young, 2018: Stimulated generation: Extraction of energy from balanced flow by nearinertial waves. J. Fluid Mech., 847, 417-451, https://doi.org/ 10.1017/jfm.2018.308.

Salmon, R., 1998: Lectures on Geophysical Fluid Dynamics. Oxford University Press, $400 \mathrm{pp}$.

- 2016: Variational treatment of inertia-gravity waves interacting with a quasi-geostrophic mean flow. J. Fluid Mech., 809, 502-529, https://doi.org/10.1017/jfm.2016.693.

Smith, K. S., and E. Bernard, 2013: Geostrophic turbulence near rapid changes in stratification. Phys. Fluids, 25, 046601, https:// doi.org/10.1063/1.4799470.

Soares, S., and K. Richards, 2013: Radiation of inertial kinetic energy as near-inertial waves forced by tropical Pacific easterly waves. Geophys. Res. Lett., 40, 1760-1765, https:// doi.org/10.1002/grl.50387.

Thomson, R. E., and W. S. Huggett, 1981: Wind-driven inertial oscillations of large spatial coherence. Atmos.-Ocean, 19, 281306, https://doi.org/10.1080/07055900.1981.9649116.

Tulloch, R., and K. Smith, 2006: A theory for the atmospheric energy spectrum: Depth-limited temperature anomalies at the tropopause. Proc. Natl. Acad. Sci. USA, 103, 14690-14694, https://doi.org/10.1073/pnas.0605494103.

Vallis, G. K., 2017: Atmospheric and Oceanic Fluid Dynamics. Cambridge University Press, 738 pp.

Van Meurs, P., 1998: Interactions between near-inertial mixed layer currents and the mesoscale: The importance of spatial variabilities in the vorticity field. J. Phys. Oceanogr., 28, 1363-1388, https://doi.org/10.1175/1520-0485(1998)028<1363: IBNIML $>2.0 . \mathrm{CO} ; 2$.

Wagner, G. L., and W. R. Young, 2015: Available potential vorticity and wave-averaged quasi-geostrophic flow. J. Fluid Mech., 785, 401-424, https://doi.org/10.1017/jfm.2015.626. 
and -2016 : A three-component model for the coupled evolution of near-inertial waves, quasi-geostrophic flow and the near-inertial second harmonic. J. Fluid Mech., 802, 806837, https://doi.org/10.1017/jfm.2016.487.

Webster, F., 1968: Observations of inertial-period motions in the deep sea. Rev. Geophys., 6, 473-490, https://doi.org/10.1029/ RG006i004p00473.

Weller, R. A., 1985: Near-surface velocity variability at inertial and subinertial frequencies in the vicinity of the California Current. J. Phys. Oceanogr., 15, 372-385, https://doi.org/ 10.1175/1520-0485(1985)015<0372:NSVVAI > 2.0.CO;2.

Whalen, C., L. Talley, and J. MacKinnon, 2012: Spatial and temporal variability of global ocean mixing inferred from Argo profiles. Geophys. Res. Lett., 39, L18612, https://doi.org/ 10.1029/2012GL053196.

, J. MacKinnon, and L. Talley, 2018: Large-scale impacts of the mesoscale environment on mixing from wind-driven internal waves. Nat. Geosci., 11, 842-847, https://doi.org/ 10.1038/s41561-018-0213-6.

Xie, J.-H., and J. Vanneste, 2015: A generalised-Lagrangianmean model of the interactions between near-inertial waves and mean flow. J. Fluid Mech., 774, 143-169, https://doi.org/ 10.1017/jfm.2015.251.

Young, W. R., and M. Ben Jelloul, 1997: Propagation of nearinertial oscillations through a geostrophic flow. J. Mar. Res., 55, 735-766, https://doi.org/10.1357/0022240973224283.

Zhai, X., R. J. Greatbatch, and J. Zhao, 2005: Enhanced vertical propagation of storm-induced near-inertial energy in an eddying ocean channel model. Geophys. Res. Lett., 32, L18602, https://doi.org/10.1029/2005GL023643.

, - C C. Eden, and T. Hibiya, 2009: On the loss of windinduced near-inertial energy to turbulent mixing in the upper ocean. J. Phys. Oceanogr., 39, 3040-3045, https://doi.org/ 10.1175/2009JPO4259.1. 